\title{
Variations in the ratio of working-memory to reference-memory trials reveal sample-encoding effects in pigeon short-term memory
}

\author{
ANGELO SANTI, WILLIAM REASON, COLLEEN HANEMAAYER, and SUSAN PETELKA \\ Wilfrid Laurier University, Waterloo, Ontario, Canada
}

\begin{abstract}
Two groups of pigeons received training on a delayed conditional discrimination [workingmemory (WM) trials] and a simple simultaneous discrimination [reference-memory (RM) trials]. For the same-sample group, the nominal sample stimuli on RM trials were the same as the sample stimuli on WM trials. For the different-sample group, they were different. In Experiment 1, the ratio of WM to RM trials $(1: 5,1: 1$, or 5:1) and the delay interval $(1,4$, or $8 \mathrm{sec})$ were manipulated. WM trial accuracy decreased as the ratio of WM to RM trials increased for the differentsample group, but variations in this ratio had little effect in the same-sample group. In Experiment 2, variations in the ratio of WM trials to either RM trials or unfilled time intervals (UTIs) approximately equivalent to the duration of an RM trial were studied. When WM trials occurred in the context of RM trials, variations in the trial ratios produced the same results as in Experiment 1. Substituting UTIs for RM trials had a much greater effect on the performance of the same-sample group than on that of the different-sample group. In the same-sample group, WM trial accuracy was affected by variations in the ratio of WM trials to UTIs but not by variations in WM-to-RM trial ratios. These effects appear to be mediated by changes in sample-encoding processes, rather than by intrusions from previous choices or previous samples.
\end{abstract}

The performance deficits observed when matching-tosample trials are closely spaced has been attributed to two separate factors: (1) a general interference effect associated with decreases in ITI, and (2) intrusion of information from previous trials. These intrusions can consist of previously presented samples or previous choices. Roitblat and Scopatz (1983) have identified intrusions from previous choices as being much more important than intrusions from previous samples. Edhouse and White (1988) have also shown that although the sample presented on trial $\mathrm{N}-1$ influences trial $\mathrm{N}$ performance, the major influence on trial $\mathrm{N}$ performance is the choice made on trial $N-1$. In addition, several studies have shown that interference due to the prior sample or the prior choice does not vary as a function of ITI duration (Edhouse \& White, 1988; Roberts, 1980; Roitblat \& Scopatz, 1983).

In a study of the effects of scopolamine on working and reference memory in pigeons, Santi, Hanemaayer, and Reason (1987) utilized a delayed conditional discrimination as the working-memory (WM) task, and a simple simultaneous discrimination as the reference-memory

This research was supported by Grant A6378 from the Natural Sciences and Engineering Research Council of Canada to Angelo Santi. Portions of this paper were presented at the annual meeting of the Canadian Psychological Association, Toronto, Ontario, Canada in June 1986. Reprints may be obtained from Angelo Santi, Department of Psychology, Wilfrid Laurier University, Waterloo, Ontario N2L 3C5, Canada.
(RM) task. On WM trials, the correct response was to select the color-comparison stimulus that matched the previously presented red or green sample. On RM trials, line tilts were presented as comparisons. The correct response was to select one particular line tilt regardless of the sample stimulus presented on that trial. Two groups of birds received training on these tasks. For one group, the same-sample group, the nominal sample stimuli on RM trials were the same as the sample stimuli on WM trials-that is, red and green colors. The other group of birds, the different-sample group, were trained with a circle and a triangle as nominal samples on RM trials and with red and green colors as samples on WM trials. Although both groups performed equally well on RM trials, the different-sample group had a much higher level of accuracy on WM trials. The same results were also observed by Santi, Bogles, and Petelka (1988).

This difference cannot be attributed to greater intrusions from previous choices for the same-sample group, because the comparison-presentation phase of each trial was identical for the two groups. The difference in accuracy between these two groups could be due to greater interference from the prior sample in the same-sample group, to a general sample-encoding deficit, or to a combination of these two effects. The purpose of the present research was to examine the WM performance differences between the same-sample and the different-sample groups in terms of these factors. 


\section{EXPERIMENT 1}

In Experiment 1, both groups received test sessions in which the ratio of WM to RM trials was manipulated such that it was $1: 5,1: 1$, or $5: 1$. As the trial ratio increases for the different-sample group, the opportunity for interference from the previous sample increases. For the samesample group, interference on WM trials from the previous sample remains constant as the trial ratio varies, because the same events serve as samples on WM and RM trials. A consideration of this factor leads to the prediction that increasing the WM-to-RM trial ratio will produce a decrement in accuracy for the different-sample group, but will have no effect on accuracy for the same-sample group.

\section{Method}

Subjects. Thirteen White Carneaux pigeons, maintained at $80 \% \pm 20 \mathrm{~g}$ of their ad libitum weight and housed individually with constant access to grit and water, served as subjects. All birds had extensive prior experience with this delayed matching-to-sample task (Santi et al., 1988; Santi et al., 1987).

Apparatus. Four Coulbourn modular operant test cages (Model E1010), housed individually in isolation cubicles (Model E10-20), were used. Each cage was equipped with three horizontally aligned, clear plastic keys behind which projectors could display the stimuli (a red or green field, a $45^{\circ}$ or $135^{\circ}$ white line on a black background, a white circle or white triangle on a black background, or a black dot on a white background) onto a frosted rear projection screen (Coulbourn Model E2118). Directly below the center key was a $5.7 \times 5.0 \mathrm{~cm}$ opening that provided access to a hopper filled with mixed grain (Coulbourn Model E14-10). All experimental events and response measures were arranged and recorded by a microcomputer system located in an adjacent room.

Procedure. All birds had received training in a delayed matching procedure in which each trial began with illumination of the center key by a warning stimulus (a black dot on a white background). A peck on the warning stimulus was followed by the presentation of either a red or a green sample stimulus on WM trials. On RM trials, the nominal sample stimulus was red or green for the 6 birds in the same-sample group, and a triangle or a circle for the 7 birds in the different-sample group. The sample stimulus was terminated after $5 \mathrm{sec}$ and was followed immediately by the presentation of the comparison stimuli on the side keys. On WM trials, the correct response was to choose the comparison that matched the previously presented sample. On RM trials, a response to the $45^{\circ}$ line was correct for 4 birds in the same-sample group and for 3 birds in the different-sample group. A response to the $135^{\circ}$ line was correct for the remaining birds in both groups. A response to a side key on both WM and RM trials terminated the comparison stimuli and resulted in either 3-sec access to mixed grain, if correct, or a 3-sec blackout, if incorrect. Following either reinforcement or blackout, an ITI of $15 \mathrm{sec}$ was spent in darkness. Each baseline training session consisted of $24 \mathrm{WM}$ trials and $24 \mathrm{RM}$ trials presented in six individually randomized blocks of the eight different combinations of sample and comparison stimuli counterbalanced for position on the side keys.

During the test phase, nine treatment conditions defined by the factorial combination of WM-to-RM trial ratio $(1: 5,1: 1$, or $5: 1)$ and de-

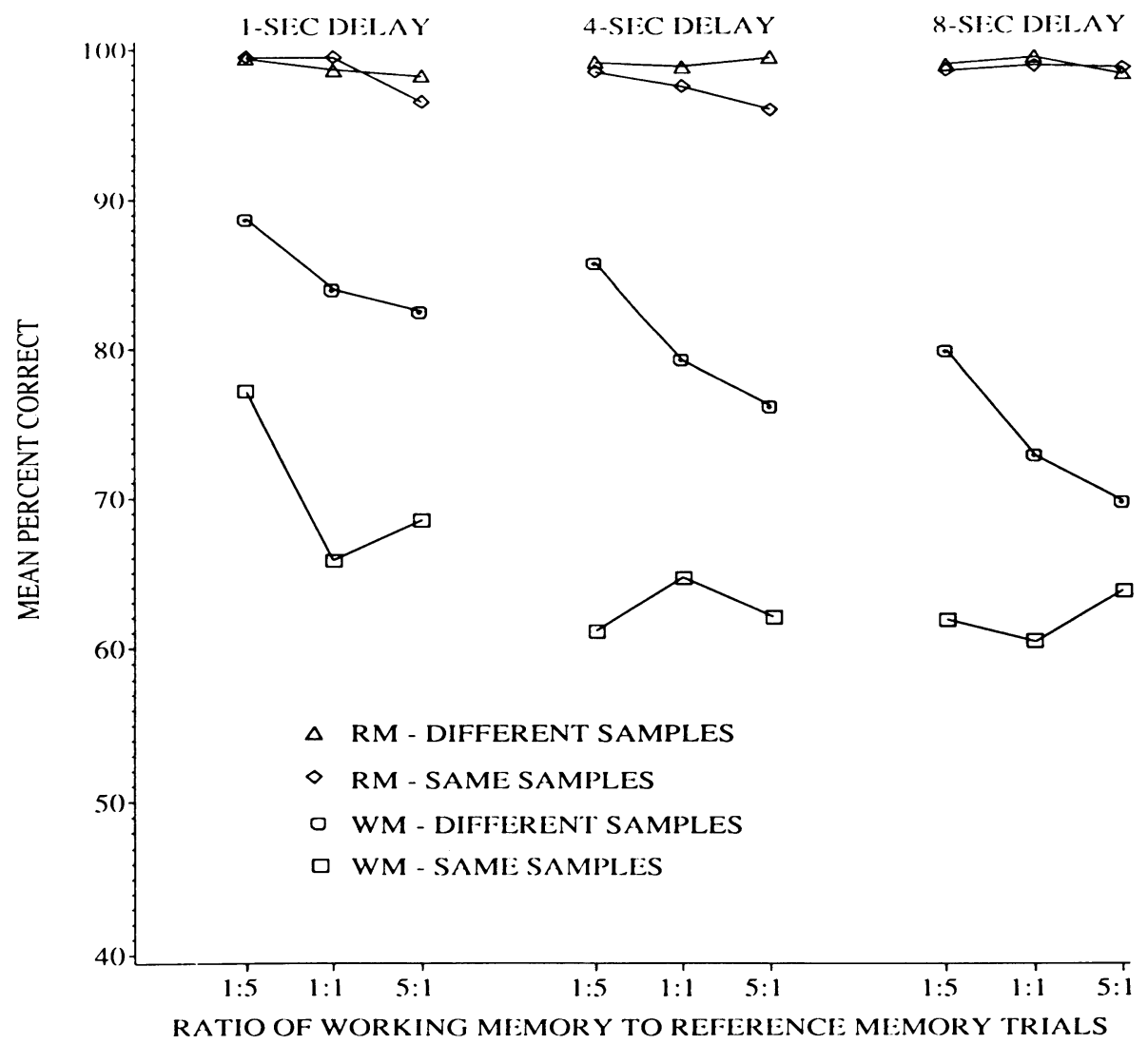

Figure 1. Mean percentage of correct responses as a function of the ratio of working-memory (WM) to reference-memory (RM) trials and delay interval in Experiment 1, for the same-sample and the different-sample groups. 
lay interval (DI) $(1,4$, or $8 \mathrm{sec})$ were presented in a randomized order. Only one treatment condition was presented on a given test day. Within each block of 9 days, each of the nine treatment conditions was tested once. Each test session consisted of 72 trials, with the trial ratios programmed in blocks of six trials. That is, within each block of six trials, the number of WM and RM trials was 1 and 5, 3 and 3, or 5 and 1 , respectively. During test sessions, the ITI was $1 \mathrm{sec}$. All other aspects of the test sessions were identical to those previously described. A total of 18 test sessions were given.

\section{Results}

The mean percentages of correct matching performance during the test sessions are shown in Figure 1. As the ratio of WM to RM trials increased, accuracy on WM trials declined significantly for the different-sample group $[F(2,22)=16.05]$. However, for the same-sample group, accuracy on WM trials was not affected by increases in the trial ratio at either the $4-$ or the 8 -sec DI $(F \mathrm{~s}<2)$. Trial ratio did not affect $\mathrm{RM}$ trial accuracy for either group. The WM performance of the different-sample group approached that of the same-sample group as the ratio of WM to RM trials increased and the DI was increased. At the 5:1 ratio and the 8-sec DI, there was no difference in WM trial accuracy between the two groups $(F<1)$.

\section{EXPERIMENT 2}

The findings of Experiment 1 suggested that the difference in accuracy between the same-sample group and the different-sample group was not due to intrusions from prior choices, but was more likely due to deficits in sample encoding or to intrusions from prior samples. One way of attempting to distinguish between this sample-encoding explanation and the prior-sample intrusion explanation is to substitute unfilled time intervals (UTIs) for RM trials. According to the sample-encoding explanation, this should facilitate the WM performance of the same-sample group much more than the WM performance of the differentsample group. The UTIs should reduce the frequency of episodes of sample encoding in the same-sample group, but it should have little or no effect in the different-sample group, because the use of different samples on RM trials already reduces the frequency of episodes of sample encoding. The prior-sample intrusion explanation does not lead to any predictions regarding this manipulation, since previous research has indicated that the strength of intrusion effects is not affected by the amount of time between trials (Edhouse \& White, 1988; Roberts, 1980; Roitblat \& Scopatz, 1983). In Experiment 2, the two groups of

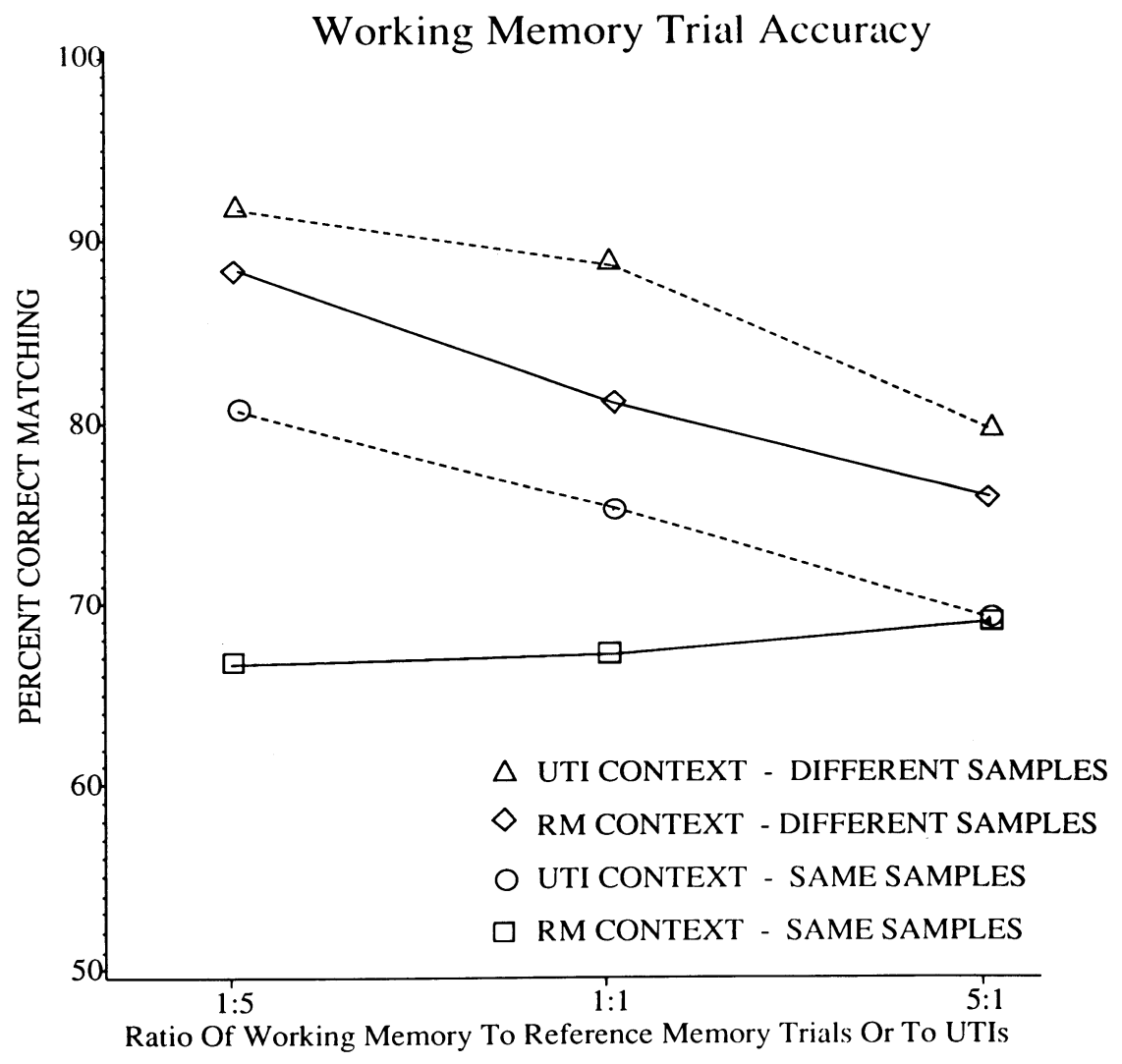

Figure 2. Mean percentage of correct responses on working-memory (WM) trials as a function of the ratio of WM trials to either reference-memory (RM) trials or unfilled time intervals (UTIs) in Experiment 2, for the same-sample and the different-sample groups. 
birds were tested with WM-to-RM trial ratios of 1:5, 1:1, or 5:1. On half of the test sessions, RM trials were intermixed with WM trials; on the other half of the test sessions, UTIs approximately equivalent to the duration of an RM trial were intermixed with WM trials at the stated ratios.

\section{Method}

Subjects and Apparatus. Thirteen subjects and the apparatus were the same as in the previous experiment. One additional bird was added to the same-sample group for this study.

Procedure. The birds received 30 sessions of baseline training following the completion of testing with the same conditions as those used during baseline training for Experiment 1 . During the test sessions, six treatment conditions, defined by the trial-ratio variable $(1: 5,1: 1$, or $5: 1)$ and whether the events intermixed with WM trials were RM trials or UTIs, were presented in a randomized order. Only one treatment condition was presented on a given test day. Within each block of 6 days, each of the six treatment conditions was tested once. Each test session consisted of 72 trials, with the trial ratios programmed in blocks of six trials as described previously. The DI during the test sessions was $4 \mathrm{sec}$, and the ITI duration was $1 \mathrm{sec}$. On test sessions in which UTIs were substituted for RM trials, the warning light appeared at the beginning of all trials. Following a peck to the warning light, the sample stimulus was presented if a WM trial had been scheduled; however, a UTI of $14 \mathrm{sec}$ was presented if an RM trial had been scheduled to occur. The duration of $14 \mathrm{sec}$ was estimated as the typical duration of an RM trial. All other aspects of the test sessions were the same as those described previously. A total of 24 test sessions were given.

\section{Results}

The mean percentages of correct matching performance on WM trials during the test sessions are shown in Figure 2. Accuracy on RM trials is not shown because it did not differ as a function of groups or trial ratios. When WM trials occurred in the context of RM trials, a variation in the trial ratio affected performance in the different-sample group $[F(2,24)=17.51]$ but not in the same-sample group $(F<1)$. When the ratio of WM to RM trials was 1:5, accuracy in the different-sample group was substantially greater than accuracy in the same-sample group $[F(1,12)=10.73]$. However, as the ratio of WM to RM trials increased, accuracy in the different-sample group declined such that, at the 5:1 ratio, there was no significant difference between the two groups $[F(1,12)=$ 1.04]. In addition, substituting UTIs for RM trials had a significant effect on the performance of the same-sample group $[F(1,12)=11.33]$, but not on that of the differentsample group $(F<1)$ at the $1: 5$ ratio.

\section{DISCUSSION}

The present research was designed to assess the validity of the sampleencoding deficit explanation of WM performance differences between a group that received different samples on WM and RM trials and a group that received the same samples on these two trial types. Interference from the prior sample can account for the finding that a variation in the ratio of WM to RM trials affected the performance of the differentsample group but not that of the same-sample group. However, this factor cannot account for the effects associated with variations in the ratio of WM trials to UTIs. For both groups, accuracy increased as the ratio of WM trials to UTIs decreased. Variations in this ratio essentially can be viewed as a manipulation of ITI duration. The smaller the ratio of WM trials to UTIs, the longer the time interval between WM trials. Previous studies (Edhouse \& White, 1988; Roberts, 1980; Roitblat \& Scopatz, 1983) have shown that interference due to the prior sample or the prior choice does not interact with ITI duration. Consequently, variations in ITI duration are viewed as producing "general" interference effects, which are independent of the effects associated with events from prior trials. This general interference has been identified with a sample-encoding deficit (Roberts, 1980) or with fatigue of a dimensionspecific analyzer (Roitblat, 1984). It would appear that the differences in accuracy on WM trials between the same-sample and the differentsample groups, as well as the effects associated with variations in the ratio of WM trials to either RM trials or UTIs in both groups, are to a great extent the result of variations in sample-encoding processes, rather than intrusions from previous choices or previous samples.

\section{REFERENCES}

Edhouse, W. V., \& White, K. G. (1988). Sources of proactive interference in animal memory. Journal of Experimental Psychology: Animal Behavior Processes, 14, 56-70.

ROBERTS, W. A. (1980). Distribution of trials and intertrial retention in delayed matching-to-sample with pigeons. Journal of Experimental Psychology: Animal Behavior Processes, 6, 217-237.

RoITBLAT, H. L. (1984). Representations in pigeon working memory. In H. L. Roitblat, T. G. Bever, \& H. S. Terrace (Eds.), Animal cognition (pp. 79-98). Hillsdale, NJ: Erlbaum.

RoItblat, H. L., \& SCOPATZ, R. A. (1983). Sequential effects in pigeon delayed matching-to-sample performance. Journal of Experimental Psychology: Animal Behavior Processes, 9, 202-221.

Santi, A., Bogles, J., \& Petelka, S. (1988). The effect of scopolamine and physostigmine on working and reference memory in pigeons. Behavioral \& Neural Biology, 49, 61-73.

Santi, A., Hanemaayer, C., \& Rason, W. (1987). The effect of scopolamine on reference and working memory in pigeons. Animal Learning \& Behavior, 15, 395-402.

(Manuscript received June 30, 1989.) 